\title{
The Paired-Electron Crystal in the Two-Dimensional Frustrated Quarter-Filled Band
}

\author{
H. Li ${ }^{1}$, R. T. Clay $^{2}$, and S. Mazumdar ${ }^{1}$ \\ ${ }^{1}$ Department of Physics, University of Arizona, Tucson, AZ 85721 \\ ${ }^{2}$ Department of Physics and Astronomy and $\mathrm{HPC}^{2}$ Center for Computational \\ Sciences, Mississippi State University, Mississippi State MS 39762
}

\begin{abstract}
The competition between antiferromagnetic and spin-singlet ground states within quantum spin models and the $\frac{1}{2}$-filled band Hubbard model has received intense scrutiny. Here we demonstrate a frustration-induced transition from Néel antiferromagnetism to spin-singlet in the interacting $\frac{1}{4}$-filled band on an anisotropic triangular lattice. While the antiferromagnetic state has equal charge densities 0.5 on all sites, the spin-singlet state is a paired-electron crystal, with pairs of charge-rich sites separated by pairs of charge-poor sites. The paired-electron crystal provides a natural description of the spin-gapped state proximate to superconductivity in many organic charge-transfer solids. Pressure-induced superconductivity in these correlated-electron systems is likely a transition from the $\frac{1}{4}$-filled band valence bond solid to a valence bond liquid.
\end{abstract}

PACS numbers: 71.10.Fd, 71.10.Hf, 74.20.Mn, 74.70.Kn

Submitted to: J. Phys.: Condens. Matter 
Quasi-two-dimensionality, strong electron-electron repulsion, and proximity of the superconducting state to semiconducting states with spatial broken symmetry are the common features between superconducting cuprates and organic charge transfer solids (CTS). Superconductivity in the CTS is reached from the semiconducting state by the application of pressure at constant carrier density. In the $\kappa$-(BEDT-TTF $)_{2} \mathrm{X},(\kappa$ $(\mathrm{ET})_{2} \mathrm{X}$ ), the CTS family with the highest $\mathrm{T}_{c}$, dimers of BEDT-TTF (ET) molecules form an anisotropic triangular lattice. Carrier concentration of one hole per dimer and commensurate antiferromagnetism [1] prompted theoretical description of these CTS within the $\frac{1}{2}$-filled band Hubbard model $[1,2]$ at ambient pressure. Within mean-field theories, pressure enhances spin frustration, destroys antiferromagnetism, and leads to superconductivity over a range of anisotropy (see reference [3] for a review). This viewpoint has been challenged by numerical calculations that demonstrate the absence of superconductivity within the triangular-lattice $\frac{1}{2}$-filled band Hubbard model for all $U$ and anisotropy [4-6]. Recent experiments also find a puzzling array of broken symmetries different from the antiferromagnetic order in the semiconducting state proximate to superconductivity in the CTS, including spin-gap [7-10], charge-ordering $[7,8]$, and coexisting charge-ordering and spin-gap [8,11]. The close relationships between the molecular and crystal structures of the two-dimensional (2D) CTS with different semiconducting phases indicate the need for a common theoretical description that can explain this panoply of competing and coexisting orders. We present here such a unified theoretical description: we show that a single structural parameter, the extent of geometrical lattice frustration - determines the nature of the dominant broken symmetry. We develop the concept of the paired-electron crystal (PEC), which provides a new paradigm for spin-singlet formation in dimensionality $>1$, and perhaps also the insight to understanding unconventional superconductivity in the CTS and related $\frac{1}{4}$-filled band inorganic materials. We point out that concept of a PEC driven by intra-pair quantum exchange occurring at intermediate densities in the electron gas has previously been postulated [12]. The mechanism of PEC formation that we demonstrate here is related, with the difference that our results apply to a lattice, with specific carrier concentration.

The idea that frustration-induced quantum effects can drive a transition from antiferromagnetism to a resonating valence bond spin-singlet state [13] has led to many exciting developments in the theory of correlated-electron systems. With few exceptions [14], however, the consequences of lattice frustration have been investigated mostly for the $\frac{1}{2}$-filled band Hubbard model, which for large $U$ reduces to an antiferromagnetic spin Hamiltonian. The effective $\frac{1}{2}$-filled band model for the 2D CTS precludes charge order and clearly cannot be the basis of the unifying theoretical description we seek. The number of carriers $n$ per molecule of the CTS is $\frac{1}{2}$. Here we focus on this specific $n$ and investigate the consequences of lattice frustration within an electronic model with simultaneous charge- and spin-frustration.

Before proceeding to $2 \mathrm{D}$, it is instructive to summarize the behavior of the $n=\frac{1}{2}$ strongly correlated band in one- and quasi one-dimension, (1D and quasi-1D), where 

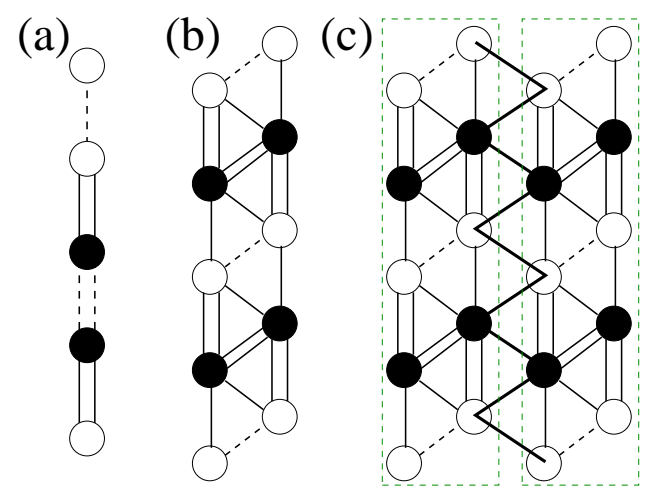

Figure 1. (color online) (a) The PEC in the one dimensional $\frac{1}{4}$-filled band spin-Peierls state. Here, and in the following, filled (empty) circles correspond to sites with a charge density of $0.5+\delta(0.5-\delta)$. Double lines are intra-dimer bonds; the inter-dimer bonds alternate between strong (double-dotted) and weak (dotted). The double-dotted bond is a localized singlet [15]. (b) PEC state in the zigzag ladder lattice. Singlets are formed between the two chains [16]. (c) Conceptualization of the 2D PEC emerging from the coupling of zigzag ladders.

also quantum effects are strong. In 1D chains, a metal-insulator transition occurs at intermediate temperatures to either a bond-dimerized state with equal site charge densities, or a charge-ordered Wigner crystal with equal intermolecular bond distances [15]. For strong enough electron-phonon interactions, and not too strong nearestneighbor electron-electron repulsion, a spin-Peierls transition occurs within both the insulating phases. The spin-Peierls state can be thought of as further bond-dimerization of the bond-dimerized state with schematic charge occupancies $\cdots 1100 \cdots[15]$, where ' 1 ' and ' 0 ' denote charge-rich and charge-poor sites. The interdimer 1-1 bond, stronger than the $0-0$ bond, constitutes a localized singlet bond (see Fig. 1(a)). This structure is the simplest example of the PEC and is observed in many 1D CTS [15]. Coexistence of charge-order and spin-gap is a consequence of co-operation between electron-phonon and nearest-neighbor antiferromagnetic spin interactions. This quantum effect dominates for a wide range of parameters over the classical effect due to the nearest-neighbor Coulomb repulsion, which prefers the Wigner crystal configuration $\cdots 1010 \cdots[15]$.

There also exist "ladder" CTS with isolated pairs of $n=\frac{1}{2}$ chains [17]. The low temperature spin-gap state found in these quasi-1D materials can be explained as a PEC in a two-leg zigzag ladder lattice, with interchain localized singlet bonds (see Fig. 1(b)) The PEC occurs when the frustrating interchain hopping exceeds a critical value [16]. Interestingly, in both the $n=\frac{1}{2} 1 \mathrm{D}$ chain and the zigzag ladder, the PECs can be thought to be derived from the corresponding $n=1$ valence bond solids by simply replacing alternate spin-singlet bonds with pairs of vacancies. Coupling $n=1$ zigzag ladders to generate the triangular lattice necessarily leads to the loss of the valence bond solid structure [13]. As shown in Fig. 1(c), however, similar coupling of the $n=\frac{1}{2}$ zigzag ladders can in principle lead to a 2D PEC with localized spin-singlet bonds. We demonstrate below that this is exactly what happens in $2 \mathrm{D}$ for large enough 


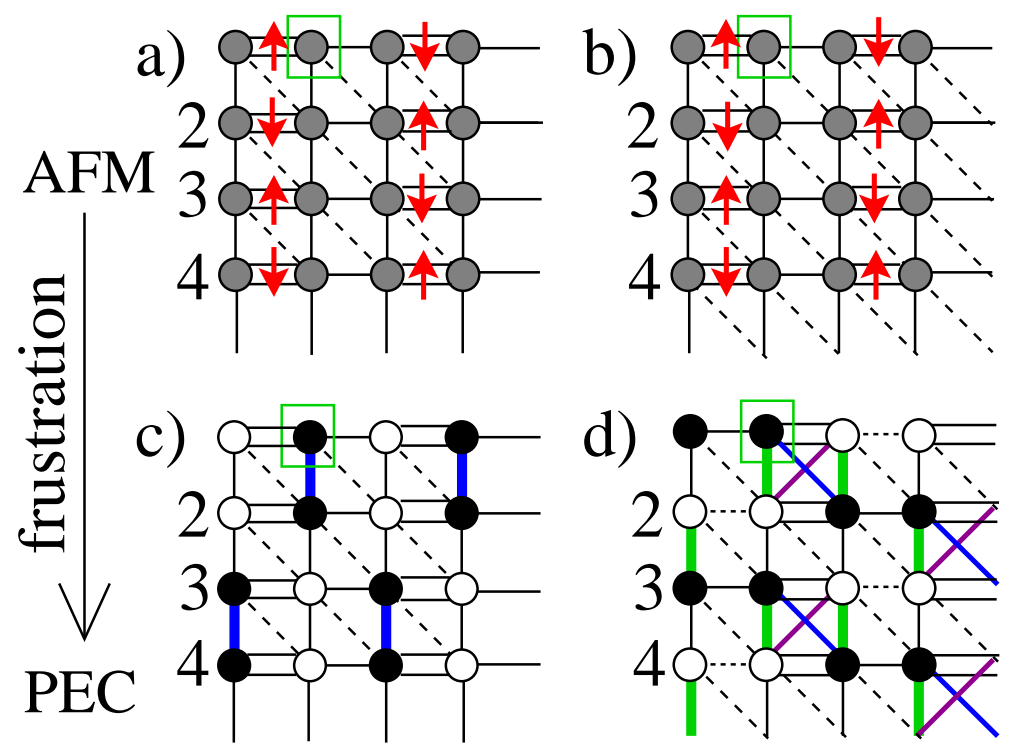

Figure 2. (color online) (a) OBC and (b) PBC $4 \times 4$ lattices for $t^{\prime}<t_{c}^{\prime}$. Double bonds and thick lines imply strong bonds; thin lines imply weak bonds. The dashed lines are the diagonal bonds whose strength is varied. Charge densities are uniform as indicated by grey circles and spin ordering corresponds to antiferromagnetism (AFM). (c) and (d) show the PEC state occuring for $t^{\prime}>t_{c}^{\prime}$. Black and white circles represent charge-rich and charge-poor sites. Singlet bonds form between the charge-rich sites in the PEC. Numbers correspond to the chain indices in Fig. 3(a)-(b). Boxes mark site 2 of chain 1. Spin-spin correlations between this site and sites on other chains are shown in Fig. 3.

lattice frustration. Furthermore, the PEC with localized singlet valence bonds between the charge-rich sites necessarily has a spin-gap, and thus a transition occurs from the antiferromagnetic to the spin-gapped state in $\frac{1}{4}$-filled $2 \mathrm{D}$ systems with increasing lattice frustration.

We consider the $\frac{1}{4}$-filled extended Hubbard model on an anisotropic triangular lattice,

$$
\begin{aligned}
& H=-\sum_{\nu,\langle i j\rangle_{\nu}} t_{\nu}\left(1+\alpha_{\nu} \Delta_{i j}\right) B_{i j}+\frac{1}{2} \sum_{\nu,\langle i j\rangle_{\nu}} K_{\alpha}^{\nu} \Delta_{i j}^{2} \\
& +\beta \sum_{i} v_{i} n_{i}+\frac{K_{\beta}}{2} \sum_{i} v_{i}^{2}+U \sum_{i} n_{i \uparrow} n_{i \downarrow}+\frac{1}{2} \sum_{\langle i j\rangle} V_{i j} n_{i} n_{j} .
\end{aligned}
$$

In Eq. 1, $\nu$ runs over three lattice directions $(\nu=x, y, x-y$, see Fig. 2). We have considered $0.5 \leq t_{y} \leq t_{x}$, but report results here for $t_{x}=t_{y}=t$ only. In what follows, all energies are in units of $t$. Our calculations are for frustrated lattices $0 \leq t_{x-y} \equiv t^{\prime} \leq 1$. Our results for negative $t^{\prime}$ are qualitatively similar and we do not report these separately. $B_{i j}=\sum_{\sigma}\left(c_{i \sigma}^{\dagger} c_{j \sigma}+\right.$ H.c. $)$ is the electron hopping between sites $i$ and $j$ with electron creation (annihilation) operators $c_{i \sigma}^{\dagger}\left(c_{i \sigma}\right) . \alpha_{\nu}$ is the inter-site electron-phonon coupling, $K_{\alpha}^{\nu}$ is the corresponding spring constant, and $\Delta_{i j}$ is the distortion of the $i-j$ bond, determined self-consistently [15]. $v_{i}$ is the intra-site phonon coordinate and $\beta$ is the 
intra-site electron-phonon coupling with corresponding spring constant $K_{\beta} . U$ and $V_{i j}$ are on-site and nearest-neighbor Coulomb interactions, respectively.

Given the complexity and detailed nature of our numerical results, we present the outcome of our calculations at the outset. We start with the square lattice $\left(t^{\prime}=0\right.$, $V_{x-y}=0$ ) limit of Eq. 1, where two different semiconducting states are possible: (i) an inphase dimerized state with all site charge densities equal, - such a state with one electron per dimer unit cell is a 2D antiferromagnet (see Fig. 2(a),(b)); (ii) and for sufficiently large $V_{x}$ and $V_{y}$, the Wigner crystal with checkerboard site occupancies. Note that the dimerized antiferromagnetic phase and the Wigner crystal are mutually exclusive. Based on the experimentally observed antiferromagnetism in weakly frustrated CTS and the observation that in all such cases the lattice is dimerized, we choose parameters that give antiferromagnetism in the square lattice limit. In Fig. 2 we present results of exact ground state calculations on $4 \times 4$ lattices with two different boundary conditions, open $(\mathrm{OBC})$ and periodic $(\mathrm{PBC})$. Both are periodic along $x$ and $y$ directions. The $\mathrm{OBC}$ is however open along the $x-y$ direction with $12 t^{\prime}$ bonds. We did not explicitly include the electron-phonon interactions in this case. Rather, we work in the limit $\alpha_{\nu}=\beta=0$; in order to have antiferromagnetic order at $t^{\prime}=0$ here we incorporate intrinsic dimerization $t_{x}=t \pm \delta_{t}$ as indicated in Fig. 2(a). We keep $t_{y}=t$ fixed and gradually increase $t^{\prime}$ from zero and monitor different order parameters that indicate the antiferromagnetism-toPEC transition (see below). In contrast to the $\mathrm{OBC}$, the $\mathrm{PBC}$ is periodic along $x-y$ and has $16 t^{\prime}$ bonds. We now work with nonzero $\alpha_{\nu}$ and $\beta$, and all bond distortions $\Delta_{i j}$ and intra-site electron-phonon coordinates $v_{i}$ are obtained self-consistently [15]. Once again we keep $t_{y}$ fixed and increase $t^{\prime}$ from zero, but now calculate all bond distortions, charge densities and spin-spin correlations self-consistently. Importantly, the bond alternation along the $x$-axis, as seen in Fig. 2(b), and the resultant antiferromagnetism, appear now as a consequence of the self-consistent calculation.

In Fig. 3 we give numerical data for $\mathrm{OBC}$ and $\mathrm{PBC}$ lattices with Eq. 1 parameters $U=4, V_{x}=V_{y}=1, V_{x-y}=0$. For the OBC lattice $\delta_{t}=0.2$ and $\alpha_{\nu}=\beta=0$, and for the PBC $\alpha_{x}=1.3, \alpha_{y}=1.0, K_{\alpha}^{x}=K_{\alpha}^{y}=2, \beta=0.1$, and $K_{\beta}=2$. Our calculations indicate that the charge-densities are uniform for both the OBC and PBC lattices for $t^{\prime}<t_{c}^{\prime}$, where the lattices are antiferromagnetic. For $t^{\prime}>t_{c}^{\prime}$, the charge densities are nonuniform and the antiferromagnetism has disappeared. Spin-singlet formation is indicated by the large increases in the strengths of the vertical bond indicated by the thick lines in Fig. 2(c), and of the diagonal and vertical bonds indicated by the thick lines in Fig. 2(d), respectively. Below we give the details of our calculations.

In Fig. 3(a) we plot the z-z spin-spin correlation functions $\left\langle S_{2}^{z} S_{j}^{z}\right\rangle$ for $t^{\prime}=0$ between fixed site 2 of chain 1 (marked with box on first row of each lattice in Fig. 2) and sites $j$, labeled sequentially $1,2,3,4$ from the left, on neighboring chains labeled 2, 3, 4 in Fig. 2. In Fig. 3(a) only, the average spin-spin correlation with each chain has been shifted to zero in order to clearly show the antiferromagnetic pattern, which is $\cdots--++\cdots$ and $\cdots++--\cdots$, indicating Néel ordering of the dimer spin moments in both lattices. The loss of this pattern in Fig. 3(b) for large $t^{\prime}=0.7$ indicates loss 

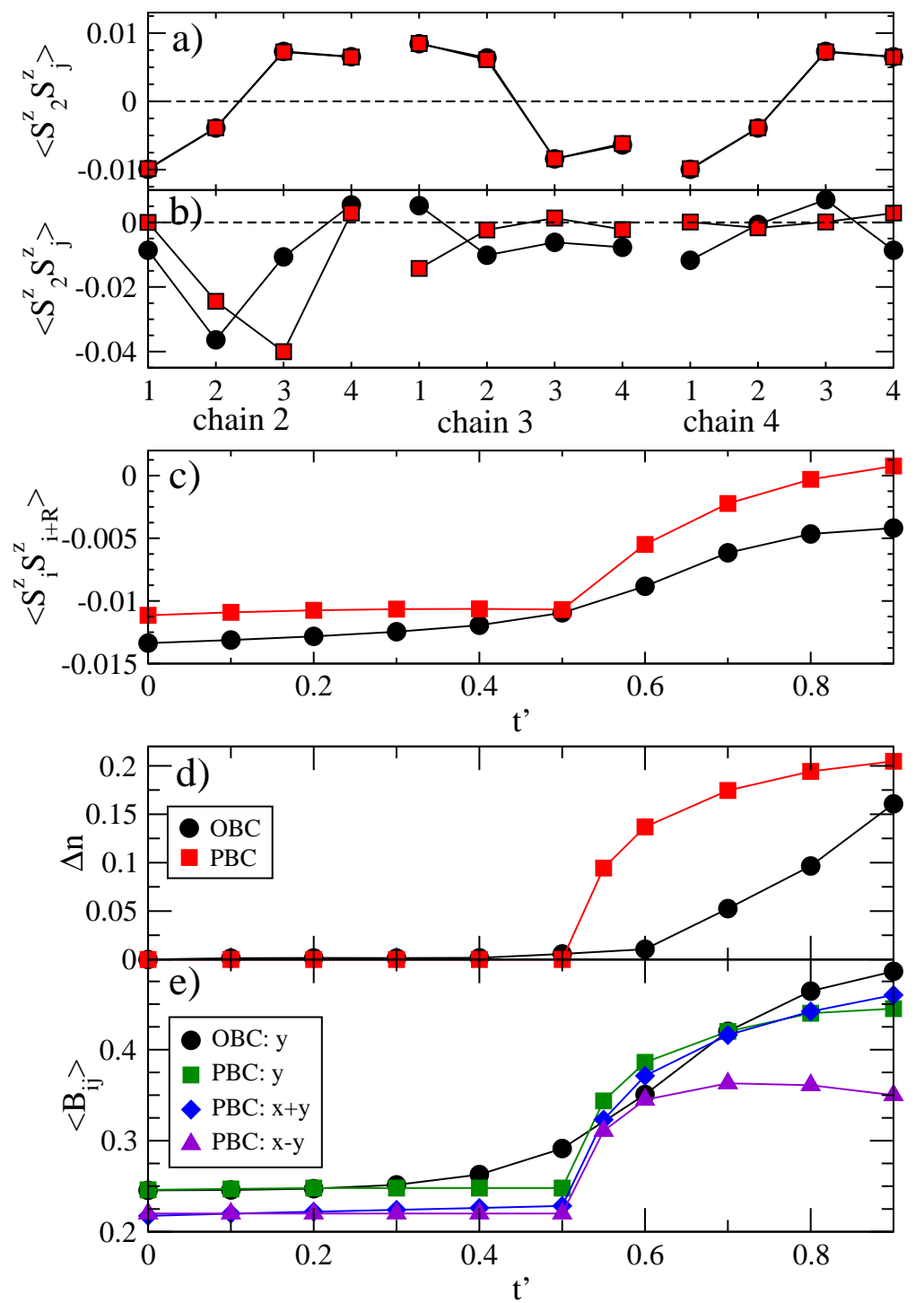

Figure 3. (color online) (a) Z-Z spin-spin correlations between site 2 in chain 1 (marked with a box in Fig. 2), and sites $1-4$ in chains 2, 3 and 4 for $t^{\prime}=0$. The chain indexing is shown in Fig. 2. In all panels, (a)-(e), circles and squares correspond to $\mathrm{OBC}$ and $\mathrm{PBC}$ calculations, respectively. (b) Spin-spin correlations as in (a), but with $t^{\prime}=0.7$. In panels (c)-(e), we plot several order parameters as a function of $t^{\prime}$. (c) Spin-spin correlations between sites that are members of the most distant dimers. (d) Difference in charge-densities between charge-rich and charge-poor sites. (e) Bondorders between pairs of nearest-neighbor sites forming localized spin-singlets. For the OBC lattice, these bonds are along the $y$ direction (Fig. 2(c)). For the PBC lattice, bonds along $y, x+y$, and $x-y$ all change at the PEC transition (see Fig. 2(d)); we plot these using squares, diamonds, and triangles, respectively. 
of antiferromagnetic order. In Fig. 3(c) we plot the z-z spin-spin correlation $\left\langle S_{i}^{z} S_{i+R}^{z}\right\rangle$ between maximally separated dimers at location $i$ and $i+R$, which measures the strength of the antiferromagnetic moment. This correlation is nearly constant and negative (as expected for the antiferromagnetic ground state, see Fig. 2(a) and (b)) until $t_{c}^{\prime} \sim 0.5$, beyond which the antiferromagnetic order is destroyed.

We define $\Delta n$ as the charge-density difference between charge-rich and charge-poor sites. Fig. 3(d) shows the rapid increase in $\Delta n$, starting from zero, for $t^{\prime}>t_{c}^{\prime}$ with both lattices. Simultaneously with charge order, there occurs a jump in the bond orders $\left\langle B_{i j}\right\rangle$ between the sites that form the localized spin-singlets. This is shown in Fig. 3(e). These bond orders are by far the strongest in both lattices for $t^{\prime}>t_{c}^{\prime}$. The spin-spin correlation between the same pairs of sites becomes strongly negative at the same $t^{\prime}$, even as all other spin-spin correlations approach zero (Fig. 3(b)), indicating spin-singlet character of the strongest bonds. Taken together, the results of Figs. 3 give conclusive evidence for the antiferromagnetism-to-PEC transition shown in Fig. 2.

We have obtained the same antiferromagnetism-to-PEC transition for $0<U \leq 8$, $0 \leq V_{x}, V_{y}, V_{x-y} \leq 2$ with both $V_{x}=V_{y}$ and $V_{x} \neq V_{y}, 0.5 \leq t_{y} \leq t_{x}$. The Coulomb interaction parameters thus cover a broad range appropriate for 2D CTS [2, 15, 18]. As we discuss below, the hopping parameters are also relevant for 2D CTS with strong frustration. Here we emphasize that although our Hamiltonian has many different parameters, in each case we fix all parameters corresponding to an antiferromagnetic state, and vary a single parameter $t^{\prime}$ to get the transition to the PEC. Thus in all cases the transition is driven by the frustration alone.

We performed several checks on our calculations to verify that the antiferromagnetism-to-PEC transition we find is not the result of finite-size effects. Possible finite size effects are usually related to (a) large discrete energy gaps in the excitation spectrum, or (b) degeneracies in the electron occupancy schemes in the $U=V_{i j}=0$ limit. Had our results been a consequence of (a), the energy gaps would have shown a sudden change at $t_{c}^{\prime}$, and this should have been visible also in the $U=V_{i j}=0$ limit. The energy gaps between the one-electron levels of our lattices change monotonically as a function of $t^{\prime}$, and there is no sudden change at $t_{c}^{\prime}$; we therefore discount possibility (a). The one-electron occupancy scheme is degenerate for all $t^{\prime}$ for the PBC, and nondegenerate for all $0<t^{\prime}<1$ for the OBC. The identical results obtained with the $\mathrm{PBC}$ and $\mathrm{OBC}$, in spite of this difference, as well as the same one-electron degeneracies for both $t^{\prime}<t_{c}^{\prime}$ and $t^{\prime}>t_{c}^{\prime}$, indicate that the transition is not associated with lattice distortions peculiar to finite systems (such as Jahn-Teller distortions). While we have shown here the results for two different boundary conditions only, we have found the same antiferromagnetism-to-PEC transition in yet another lattice, with open boundaries in both $y$ and $x-y$ directions. The complete absence of band structure-related effects, identical results obtained with three different boundary conditions, and the wide range of parameters over which we found the antiferromagnetism-to-PEC transition, taken together, give us confidence that the transition we observe is real.

We believe that there is a relatively simple physical reason for the antiferromagnet- 


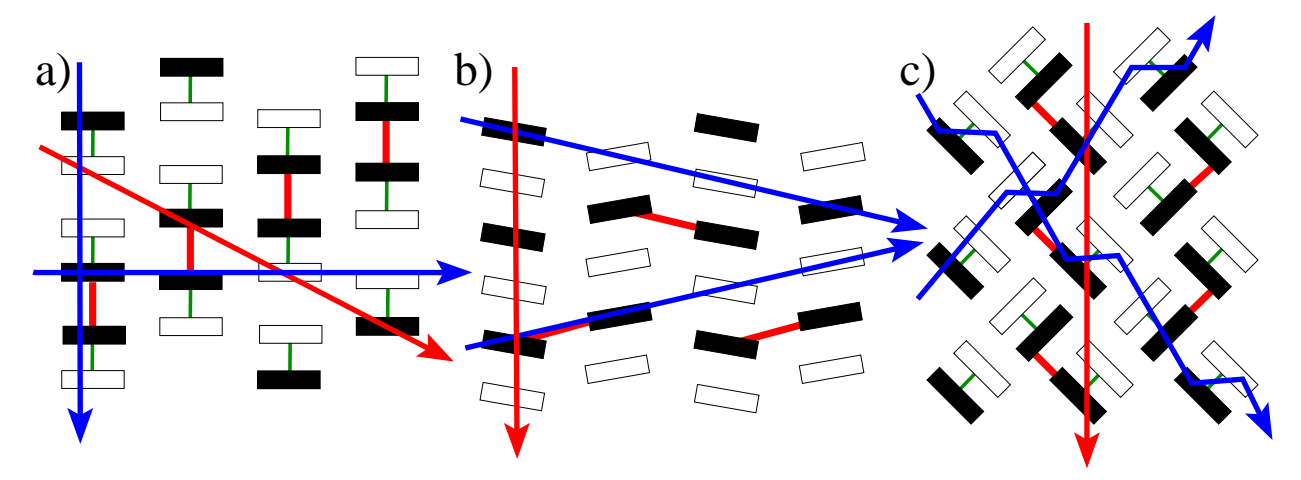

Figure 4. (color online) PEC structures for (a) $\mathrm{EtMe}_{3} \mathrm{P}\left[\mathrm{Pd}(\mathrm{dmit})_{2}\right]_{2}[11]$, and (b) $\theta$-(ET) $)_{2} \operatorname{RbZn}(\mathrm{SCN})_{4}[23]$. Black and white boxes are charge-rich and charge-poor molecules. Thin bonds are strong intradimer hopping integrals and thick bonds are spin-singlet bonds. (c) Charge occupancies and singlet bonds for $\kappa$ - $(\mathrm{ET})_{2} \mathrm{Cu}_{2}(\mathrm{CN})_{3}$ in the $T=0$ limit, as suggested from extension of the PEC concept to this system. It is conceivable that the PEC here is short-range at finite temperatures. The arrows in all cases indicate charge carrier paths $\cdots 1100 \cdots$ and $\cdots 1010 \cdots$.

to-PEC transition in the $\frac{1}{4}$-filled band in 2D. As already suggested in [13], and discussed extensively also in the recent literature [19], geometric frustration strongly enhances the tendency to form spin-singlet valence bonds. In the dimerized $\frac{1}{4}$-filled band antiferromagnet such singlet bonds will occur between dimer unit cells (see Fig. 2). Focusing on individual sites within the dimer units, strong singlet bonds must occur between nearest neighbors while bonds between distant sites must be weaker, which implies a wavefunction that is a superposition of valence bond diagrams with strong bonds between nearest neighbor sites and diagrams with weaker bonds between distant sites. Such a structure in the $\frac{1}{4}$-filled band will necessarily have charge-ordering accompanied by bond distortion (see Fig. 1(c)). Yet another way to understand the exceptional stability of the PEC at $n=\frac{1}{2}$ is to recognize this as a commensurability effect. Recall that transitions driven by electron-electron interactions, from the metallic to the Mott-Hubbard semiconductor at $n=1$, or to the Wigner crystal in bipartite lattices at $n=\frac{1}{2}$, are essentially consequences of high order commensurability. The PEC with the numbers of bonded and vacant pairs of sites exactly equal at $n=\frac{1}{2}$ is also commensurate, and there is gain in exchange energy from the strong bonding between the charge-rich sites.

The PEC paradigm enables us to explain the seemingly widely different behavior in the semiconducting states of CTS that undergo transition to superconductivity under pressure. Although 2D CTS occur in different crystalline forms, it has been pointed out that they can generally be described as anisotropic triangular lattices with varying degrees of frustration $[2,7,18,20-22]$. The PEC structures in all cases are arrived at simply by noting that the charge order pattern is (i) $\cdots 1100 \cdots$ along two of three directions of the triangular lattice, and (ii) $\cdots 1010 \cdots$ in the direction of weakest hopping (see Fig. 1(c), Fig. 2(c) and (d).) In Fig. 4, we give the PEC patterns for 
$\beta^{\prime}-\mathrm{X}\left[\mathrm{Pd}(\mathrm{dmit})_{2}\right]_{2}, \theta-(\mathrm{ET})_{2} \mathrm{X}$ and $\kappa-(\mathrm{ET})_{2} \mathrm{X}$. PECs for other crystal structures can be obtained by simple extrapolations. Thus, for example, the crystal structures of $\beta, \beta^{\prime}$ and $\beta^{\prime \prime}$ salts are related [21,24,25]; similarly, the $\alpha$-phase is obtained by slight modifications of the basic $\theta$ structure motif [7,20-22]. We discuss below the individual PEC structures of Fig. 4 and specific systems that exhibit transitions to the superconducting state from a charge-ordered or a spin-gapped state (we also discuss the antiferromagnetismto-superconductivity case below). We have focused only on ground states here. The temperatures $\mathrm{T}_{S G}$ at which the spin gap opens will depend on detailed lattice structures, and could be vanishingly small in some materials.

(i) The family of $\mathrm{X}\left[\mathrm{Pd}(\mathrm{dmit})_{2}\right]_{2}$, where $\mathrm{X}$ is a monovalent cation, have the $\beta^{\prime}$ crystal structure. Systems with relatively weak frustration exhibit antiferromagnetism [26], but those with lattice structures closest to isotropic triangular, $\mathrm{X}=\mathrm{Et}_{2} \mathrm{Me}_{2} \mathrm{Sb}$, and $\mathrm{X}=\mathrm{EtMe}_{3} \mathrm{P}$ exhibit spin gaps [27]. Thus $t^{\prime}$ in these systems lie in the region where theoretically we find the PEC to dominate over the antiferromagnetism. In Fig. 4(a) we have shown our proposed PEC pattern for the semiconducting phase of $\beta^{\prime}-\mathrm{EtMe}_{3} \mathrm{P}\left[\mathrm{Pd}(\mathrm{dmit})_{2}\right]_{2}$. Note that this pattern is obtained by simple rotations of the PECs of Fig. 2, with the y-axis (x-axis) of Fig. 2(c) (Fig. 2(d)) corresponding to the intrastack axis of Fig. 4(a). The period 4 intrastack site charge densities and intermolecular distances (strong intradimer 1-0 bond, and alternating weak interdimer 1-1 bond and weaker 0-0 bond) of Fig. 4(a) have all been observed in $\mathrm{X}=\mathrm{EtMe}_{3} \mathrm{P}[11,28]$. In our model calculation, we treat molecules as point objects, but the orientation of molecules (both intra- and inter-layer) strongly affects the magnitude of the spin gap. Clearly, "parallel" orientation of molecules, as occurs in $\mathrm{X}=\mathrm{EtMe}_{3} \mathrm{P}$ (see Fig. 3b in Reference [11]) makes the formation of a strong spin-singlet bond easier, and enhances the tendency to transition to the PEC self-consistently. The favorable orientations are responsible for the high $\mathrm{T}_{S G} \sim 25 \mathrm{~K}$ here.

Like $\beta^{\prime}-\mathrm{EtMe}_{3} \mathrm{P}\left[\mathrm{Pd}(\mathrm{dmit})_{2}\right]_{2}$, the salt $\beta$-(meso-DMET $)_{2} \mathrm{PF}_{6}$ also exhibits a pressure-induced transition from charge order to superconductivity. The charge order here also has patterns $\cdots 1100 \cdots$ in two directions and $\cdots 1010 \cdots$ along the third direction, with almost the same PEC structure (see Fig. 2 in [29].)

(ii) The PEC of Fig. 4(b) explains the spin-gap phase in the $\theta-(\mathrm{ET})_{2} \mathrm{MM}^{\prime}(\mathrm{SCN})_{4}$ family [7]. The charge order and bond patterns here are again obtained from rotations of the PECs in Fig. 2, with the y-axis (x-axis) and the diagonal direction of Fig. 2c (d) corresponding to the strong diagonal hoppings in the $\theta$-lattice of Fig. 4(b). Although theoretical [18] and experimental [30] studies showed that the charge order corresponds to the "horizontal stripe" of charge-rich sites in Fig. 4(b), the mechanism of the spingap transition was not understood until now. The "zigzag" horizontal stripe here (see Fig. 4(b)) is part of a 2D lattice and a simple one dimensional spin-Peierls mechanism for the spin-gap is not satisfactory. In the $\theta$-lattice the weakest hopping is along the stack, and has the charge pattern $\cdots 1010 \cdots$. The weak intrastack hopping is about half the strong interstack hoppings [20], making the $\theta$-lattice strongly frustrated already at high temperatures. With decreasing temperature, the intrastack lattice parameter 
decreases sharply relative to the interstack lattice parameters [23]. Within our theory, the lattice contraction leads to increased intrastack hopping, and therefore increased frustration and transition to the PEC with spin-gap within our theory.

As has been noted above, the lattice structures of $\theta$ - and $\alpha$-salts are related [20-22]. The small differences between the hopping integrals in the two classes have significant effect on band structures calculated for uncorrelated charge carriers, but are of less significance in the presence of strong electron-electron interactions. Frustration is therefore expected to play a strong role also in the $\alpha-(\mathrm{ET})_{2} \mathrm{X}$. The observation that the state obtained below the metal-insulator transition temperature in $\alpha-(\mathrm{ET})_{2} \mathrm{I}_{3}$ exhibits coexisting charge-order and spin-gap [31], as opposed to antiferromagnetism, confirms this conjecture. The recent observation of phason-like modes in the charge-ordered spin-gapped state in this material confirms that the PEC state involves coupled charge, bond, and spin degrees of freedom [32].

(iii) To date, the $\kappa$-(ET) $)_{2} \mathrm{X}$ have been modeled as a triangular lattice of dimers of ET molecules. However, it is possible to consider the lattice also as a triangular lattice of monomers with hoppings between each molecule and six neighbors (see e.g. Fig. 9 in [20]). There is a fundamental similarity between the $\kappa$ - and $\theta$-structures, in that the two directions defined by the interdimer hopping integrals $p$ in [20] once again correspond to the $x$ and $y$ directions in Fig 2, with the hopping integral labeled $b 2$ in [20] taking the role of $t^{\prime}$ in our calculations. The main difference from the $\theta$-structure is the very strong dimerization which enhances the tendency to antiferromagnetism. Indeed, simultaneous strong dimerization and frustration makes the $\kappa$-(ET) ${ }_{2} \mathrm{X}$ closer to $\beta^{\prime}$-EtMe ${ }_{3} \mathrm{P}\left[\mathrm{Pd}(\mathrm{dmit})_{2}\right]_{2}$ than to the other ET-salts [33-35].

In $\kappa$ - $(\mathrm{ET})_{2} \mathrm{X}$ relative orientations of neighboring dimers are nearly perpendicular. Assuming comparable electron-phonon couplings in the $\kappa$-(ET) ${ }_{2} \mathrm{X}$ and other CTS, much stronger lattice distortion would be needed in the $\kappa-(\mathrm{ET})_{2} \mathrm{X}$ to form static singlet bonds, and any spin-gap would be very small. This is probably the reason why a direct antiferromagnetism-to-superconductivity transition occurs under pressure in most $\kappa$ $(\mathrm{ET})_{2} \mathrm{X}$. The nearly isotropic $\kappa-(\mathrm{ET})_{2} \mathrm{Cu}_{2}(\mathrm{CN})_{3}$ does not exhibit antiferromagnetism and until recently was considered to be a spin liquid [36]. Specific heat [37] and thermal conductivity [10] measurements have claimed contradictory evidences for gapless versus gapped energy spectrum here $[10,37]$. Both experiments also find evidence for a possible phase transition at $6 \mathrm{~K}$. More recently, a second-order thermodynamic phase transition near $6 \mathrm{~K}$ that is accompanied by strong anisotropic lattice effects has been demonstrated. [38]. These investigators have determined that the resultant entropy change cannot be accounted for in terms of spin degrees of freedom alone, and have suggested that charge degrees of freedom play a strong in the transition [38]. Similarly, measurements of the dielectric response have shown increasing and frequency-dependent dielectric constant below $60 \mathrm{~K}$, and possible antiferroelectric ordering of the dipoles at a transition temperature of $\sim 9 \mathrm{~K}$, which requires unequal site charges on the dimer unit cell [39]. Both of these latter experiments $[38,39]$ are in agreement with the PEC picture, although because of the relative orientations of the ET molecules the PEC could be short-ranged. 
In Fig.4(c) we have shown the proposed PEC here: the pattern of the charge-ordering is once again $\cdots 1100 \cdots$ along two paths and $\cdots 1100 \cdots$ along the third. One possible pattern for forming spin-singlet bonds is shown in Fig.4(c). With short-ranged PEC order at finite temperatures there would be tendency to form domain-walls, as suggested from the dielectric constant measurements [39]. Importantly, (i) the charge order pattern obtained using the PEC concept outlined above is identical to those proposed in [39, 40], and (ii) the absence of similar charge-disproportionation in other $\kappa$-(ET) ${ }_{2} \mathrm{X}$ with smaller frustration is explained naturally within our theory.

We conclude that the tendency to form the PEC is ubiquitous to the strongly frustrated $\frac{1}{4}$-filled band. Experimentally, increased frustration induced by pressure or chemical substitution causes antiferromagnetism-to-superconductivity [1], antiferromagnetism-to-PEC [26] or PEC-to-superconductivity [9, 29] transitions. A unified theoretical approach for strongly-correlated superconductivity in $\frac{1}{4}$-filled materials follows if it is assumed that the superconductivity is a consequence of the transition from the valence bond solids of Fig. 4 to valence bond liquids with mobile spin-singlet bonds. The singlet bonds of the $\frac{1}{4}$-filled band PEC can be visualized as effective negative- $U$ centers in a $\frac{1}{2}$-filled band [41]. We have recently shown that within such an effective $\frac{1}{2}$-filled band negative- $U$ model with repulsive interaction between the on-site pairs there occurs a first-order transition from a chargeordered state to superconductivity with increased frustration [41]. The PEC concept lies at the interface of theories emphasizing electron-electron and electron-phonon interactions: the bipolarons in the PEC are bound not by extraordinarily strong electron-phonon interactions [42,43], but by antiferromagnetic correlations. Direct transition to the superconducting state from antiferromagnetism in most $\kappa$-systems is conceivably energetically more favorable since this does not require static lattice distortion, which we have pointed out can be energetically expensive because of their crystal structures.

Interestingly, there exist other unconventional superconductors in which electronelectron interactions, frustration and $\frac{1}{4}$-filling appear all appear to play significant roles, giving support to the mechanism of superconductivity proposed by us [41]. The cobalt ions in the family of materials $\mathrm{Na}_{x} \mathrm{CoO}_{2}$, for example, form an isotropic triangular lattice, and electron-electron interactions in these are strong [44]. Superconductivity occurs only in the hydrated material $\mathrm{Na}_{x} \mathrm{CoO}_{2} \cdot y \mathrm{H}_{2} \mathrm{O}$, with the $\mathrm{H}_{2} \mathrm{O}$ layers occurring between the $\mathrm{Na}$ and Co layers. The Co valency in the hydrated superconductor has been determined to be very close to +3.5 , indicating a $\frac{1}{4}$-filled Co hole-band $\left(\frac{3}{4}\right.$-filled electron-band) $[45,46]$. Similarly, $\mathrm{LiTi}_{2} \mathrm{O}_{4}$ and $\mathrm{CuRh}_{2} \mathrm{~S}_{4}$ are superconductors with highly frustrated 3D spinel structure and effective $\frac{1}{4}$-filled $d$-bands. As with the CTS, superconductivity in these spinels is proximate to a semiconductor, as seen from the unconventional metal-insulator transition in $\mathrm{CuIr}_{2} \mathrm{~S}_{4}$ [47], which is isoelectronic with $\mathrm{CuRh}_{2} \mathrm{~S}_{4}$. The effective $\frac{1}{4}$-filled band of $\operatorname{Ir}^{3.5+}$ ions undergoes an orbitally-driven Peierls instability at the metal-insulator transition [48], with charge-ordering $\operatorname{Ir}^{4+}-\mathrm{Ir}^{4+}-\mathrm{Ir}^{3+}-\mathrm{Ir}^{3+}$ (equivalent to the $\cdots 1100 \cdots$ pattern occuring in certain directions of the PEC), and 
spin-singlet $\mathrm{Ir}^{4+}-\mathrm{Ir}^{4+}$ bonds, in spite of the 3D nature of the crystal. We believe that the charge-ordering of the 3D lattice here reflects the same tendency of the frustrating interacting $\frac{1}{4}$-filled band to form the PEC that we have demonstrated in 2D. Work is currently in progress to demonstrate the transition to superconductivity from the $\frac{1}{4}$-filled band PEC. This work was supported by the US Department of Energy grant DE-FG02-06ER46315.

\section{References}

[1] K. Kanoda. J. Phys. Soc. Jpn., 75:051007, 2006.

[2] H. Kino and H. Fukuyama. J. Phys. Soc. Jpn., 64:2726, 1995.

[3] H. Kontani. Rep. Prog. Phys., 71:026501, 2008.

[4] T. Mizusaki and M. Imada. Phys. Rev. B, 74:014421, 2006.

[5] R. T. Clay, H. Li, and S. Mazumdar. Phys. Rev. Lett., 101:166403, 2008.

[6] L. F. Tocchio, A. Parola, C. Gros, and F. Becca. Phys. Rev. B, 80:064419, 2009.

[7] H. Mori. J. Phys. Soc. Jpn., 75:051003, 2006.

[8] N. Tajima et al. J. Phys. Soc. Jpn., 75:051010, 2006.

[9] Y. Shimizu et al. Phys. Rev. Lett., 99:256403, 2007.

[10] M. Yamashita et al. Nature Phys., 5:44, 2009.

[11] M. Tamura, A. Nakao, and R. Kato. J. Phys. Soc. Jpn., 75:093701, 2006.

[12] K. Moulopoulos and N. Ashcroft. Phys. Rev. Lett., 69:2555, 1992.

[13] P. W. Anderson. Mater. Res. Bull., 8:153, 1973.

[14] D. Poilblanc, K. Penc, and N. Shannon. Phys. Rev. B, 75:220503, 2007.

[15] R. T. Clay, S. Mazumdar, and D. K. Campbell. Phys. Rev. B, 67:115121, 2003.

[16] R. T. Clay and S. Mazumdar. Phys. Rev. Lett., 94:207206, 2005.

[17] C. Rovira. Chem. Eur. J., 6:1723, 2000.

[18] R. T. Clay, S. Mazumdar, and D. K. Campbell. J. Phys. Soc. Jpn., 71:1816, 2002.

[19] L. Balents. Nature, 464:199, 2010.

[20] T. Mori, H. Mori, and S. Tanaka. Bull. Chem. Soc. Jpn., 72:179, 1999.

[21] C. Hotta. J. Phys. Soc. Jpn., 72:840, 2003.

[22] H. Fukuyama. J. Phys. Soc. Jpn., 75:051001, 2006.

[23] M. Watanabe, Y. Noda, Y. Nogami, and H. Mori. J. Phys. Soc. Jpn., 76:124602, 2007.

[24] T. Mori. Bull. Chem. Soc. Jpn., 71:2509, 1998.

[25] The $\beta^{\prime}$ and related structures in the $\mathrm{X}\left[\mathrm{Pd}(\mathrm{dmit})_{2}\right]_{2} \mathrm{CTS}$ are similar to but not identical to the $\beta^{\prime}$ structure in ET-based CTS.

[26] M. Tamura and R. Kato. Sci. Technol. Adv. Mater., 10:024304, 2009.

[27] T. Itou et al. Phys. Rev. B, 77:104413, 2008.

[28] M. Tamura et al. Chem. Phys. Lett., 411:133, 2005.

[29] S. Kimura et al. J. Am. Chem. Soc., 128:1456, 2006.

[30] M. Watanabe, Y. Noda, Y. Nogami, and H. Mori. J. Phys. Soc. Jpn., 73:116, 2004.

[31] B. Rothaemel et al. Phys. Rev. B, 34:704, 1986.

[32] T. Ivek et al. Phys. Rev. Lett., 104:206406, 2010.

[33] The frustration parameter $t^{\prime} / t$ commonly quoted for $\kappa$-(ET) $)_{2} \mathrm{X}[34,35]$ refers to the effective $t^{\prime}$ and $t$ between dimers, and is not a useful measure of the degree of frustration in the lattice of monomers.

[34] H. C. Kandpal et al. Phys. Rev. Lett., 103:067004, 2009.

[35] K. Nakamura et al. J. Phys. Soc. Jpn., 78:083710, 2009.

[36] Y. Shimizu et al. Phys. Rev. Lett., 91:107001, 2003.

[37] S. Yamashita et al. Nature Phys., 4:459, 2008.

[38] R. Manna et al. Phys. Rev. Lett., 104:016403, 2010.

[39] M. Abdel-Jawad et al. preprint http://arxiv.org/abs/1003.3902v1. 
[40] Fig. 4(c) is oriented as in [20] and is rotated by $90^{\circ}$ relative to the lattice shown in [39].

[41] S. Mazumdar and R. T. Clay. Phys. Rev. B, 77:180515(R), 2008.

[42] R. Micnas, J. Ranninger, and S. Robaszkiewicz. Rev. Mod. Phys., 62:113, 1990.

[43] A. S. Alexandrov and N. F. Mott. High Temperature Superconductors And Other Superfluids. Taylor and Francis, London, 1994.

[44] P. Limelette et al. Phys. Rev. Lett., 97:046601, 2006.

[45] H. Sakurai et al. Phys. Rev. B, 74:092502, 2006.

[46] M. Bañobre-López et al. J. Am. Chem. Soc., 131:9632, 2009.

[47] P. G. Radaelli et al. Nature, 416:155, 2002.

[48] D. I. Khomskii and T. Mizokawa. Phys. Rev. Lett., 94:156402, 2005. 